\title{
Holes and wholes: A reply to Rubin and Kanwisher
}

\author{
LIN CHEN \\ University of Science and Technology of China, Hefei, China
}

\begin{abstract}
This paper, contrary to skepticism about the human ability to perceive topological properties, addresses some commonly heard nontopological counterexplanations and provides evidence to rule out these apparent counterexamples against the topological hypothesis. Data from improved control conditions show that the reason why these counterexamples seem inconsistent with the topological hypothesis is that they fail to take due account of two factors: visibility, and organization other than physical connectedness.
\end{abstract}

The hypothesis that a primitive and general function of the visual system is the perception of global topological properties, advanced by Chen (1982), has been tested against a fairly large set of experimental data collected within a variety of approaches and paradigms in the study of perceptual organization. These data concern phenomena such as apparent motion, sensitivity to topological distinctions, the object superiority effect, configural superiority effects, effortless texture discrimination, and competing organization with several simultaneous factors. The results have consistently supported the topological hypothesis (Chen, 1983, 1985, 1986).

One of the basic experiments revealing the robust and counterintuitive influence of topological properties on early visual processing involves the stimuli shown in Figure 1. Subjects were given T-scope presentations of one of the three pairs of figures and asked if the figures were the same or different. Performance was significantly better for the ring-disk pair than for the other two. On the average, the stimulus that contained a disk and a ring was reported as "different" on $64.5 \%$ of the presentations; the stimulus that contained a disk and a square, on $43.5 \%$; and the stimulus that contained a disk and a triangle, on $38.5 \%$. The accuracy with the stimulus containing a ring was significantly better than it was with

The title of this paper is taken from a review of the author's work in The Sciences, 23, no. 6 (1983):9, by Sarah Roxer. A preliminary version was presented at the Second Academic Conference on General and Experimental Psychology, sponsored by the Chinese Psychological Society, Chengdu, October 1985. This research was supported by grants from the Chinese National Foundation of Sciences and the State Commission of Science and Technology of China and by a research fellowship from the Alexander von Humboldt Foundation. The final draft of the article was prepared while the author was a visiting scientist with Jan Drösler at the Institut für Psychologie, Universität Regensburg. I am very grateful to Paul Smolensky for valuable advice and careful review of drafts of this paper. Helpful comments came from Rumjahn Hoosain, Hans Irtel, John Spinks, Erich Weichselgartner, and Dirk Wendt. Shi Hong-xian participated in preparing and running the experiments. Requests for reprints should be sent to Lin Chen, Beijing Laboratory of Cognitive Science, University of Science and Technology of China, Graduate School of Academia Sinica, P.O. Box 3908, Beijing, China.

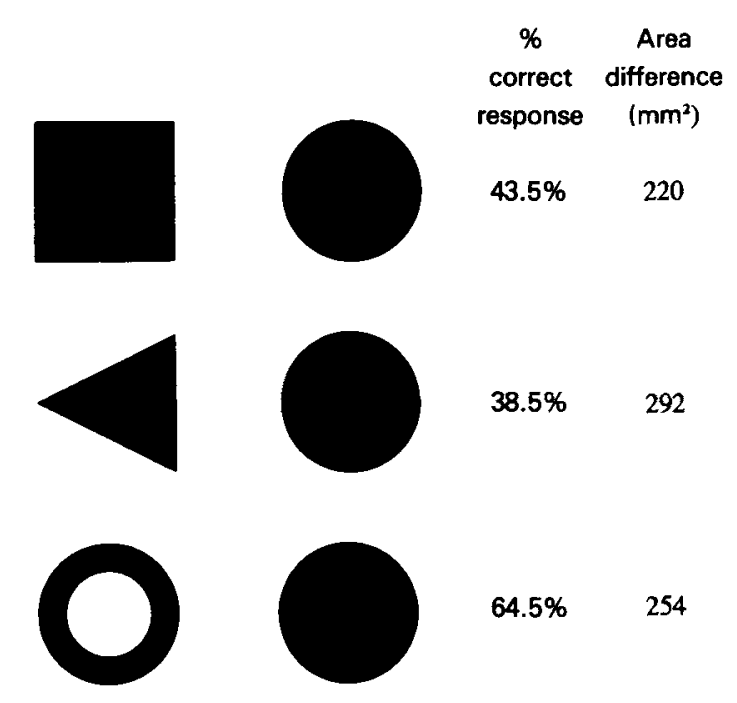

Figure 1. The three stimulus displays used in Chen's earlier experiment (adapted from Chen, 1982, Figure 1).

stimuli containing either a square or a triangle $[t(5)=$ 8.78 and 6.12 , respectively, $p<.01]$. Although an intuitive notion of similarity might lead one to expect the opposite result, topology provides an explanation of the data: The ring and disk differ topologically, in that the ring contains a hole while the disk does not. Although phenomenally they look quite different, the solid figures in the other two pairs are all topologically equivalent to each other, since any one of them can be modified to match any other by performing smooth deformation. On the other hand, smooth deformations cannot create or destroy holes, so the ring and disk are not topologically equivalent.

The topological account of this experiment (Chen, 1982) has been challenged by Rubin and Kanwisher (1985). They favor a counterexplanation commonly considered in the study of visual perception: that the results are due to differences not in topological properties but in luminous flux. They further offer new experimental results, which 
they claim are consistent with the luminous flux account and inconsistent with the topological account.

In Section 1 of this paper, I will argue that the luminous flux account fails both for Rubin and Kanwisher's (1985) data and for earlier studies reported by Chen (1982). I will further argue, in Section 2, that their new experimental results are confounded, and that when these confounds are eliminated, the results completely support the topological account. This argument depends on four experiments to be described below, two of which were performed prior to Rubin and Kanwisher's (1985) critique and two of which were a direct response to it.

It is worth observing that, like many proponents of the computational approach to vision, Rubin and Kanwisher (1985) are skeptical of the topological hypothesis because, in their words, "the determination of topological properties is not likely to be primitive for any kind of computational system"' (Rubin \& Kanwisher, 1985, p. 179). Although it would not seem to be a necessary property of computational accounts of vision, such accounts (e.g., Marr's "primal sketch"') tend to assume that the primitive elements of computation are local geometrical features. With respect to such primitives, topological properties have high computational complexity. Existing computational models, therefore, lead one to expect that discriminations based on local geometric properties would occur earlier than those based on topological propertiesexactly the opposite of the experimental results described by Chen $(1983,1985,1986)$. These data, therefore, provide a strong challenge to the computational approach, either to explain the data using local geometrical primitives, or to develop new computational models based on other primitives with respect to which topological properties have modest computational complexity. There is not yet to my knowledge any reason to believe that neural network or connectionist models will be any better suited to answer this challenge than other computational approaches. Such an answer would need to address, among other things, Minsky and Papert's (1969) classic results on the high complexity of topological properties for perceptrons. It is interesting to notice that, although, as Rubin and Kanwisher point out, "it has long been known that the primate visual system has a primitive capacity-one that survives even bilateral removal of visual cortex-to detect differences in luminous flux (Klüver, 1941)" (1985, p. 179), the experiments have suggested that topological factors may play an even more primitive role in the discriminability of form than luminous flux does.

\section{CONTROLLING FOR LUMINOUS FLUX AND OTHER NONTOPOLOGICAL EXPLANATORY FACTORS}

It is commonly argued that luminous flux differences, not topological differences (involving holes), account for experiments such as the one involving the stimuli of Figure 1.

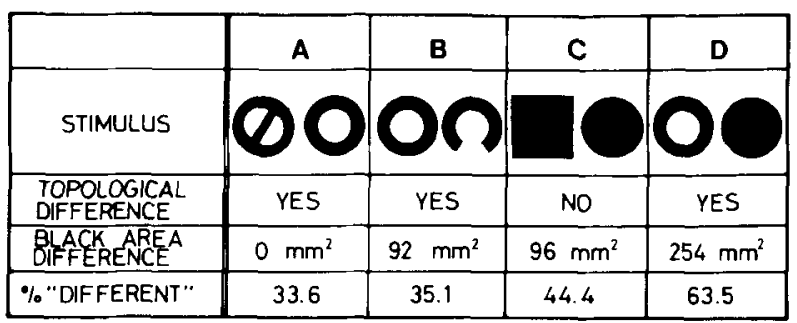

Figure 2. The four stimulus displays used in Rubin and Kanwisher's experiment, and the results (from Rubin \& Kanwisher, 1985, Figure 1).

As a matter of fact, the original experiment by Chen (1982) already controlled for luminous flux. There was no meaningful difference between the areas of the ring and the triangle, but there was a significant difference in their discriminability from the same disk. ${ }^{1}$ On the other hand, the area of the triangle was half that of the square, yet there was no significant difference in their discriminability from the same disk. (Note that the area of the triangle is obvious from Figure 1, which is a photographic reduction of the original stimuli; the dimensions of the triangle reported in Note 4 of Chen, 1982, should have been: $32 \times 35.8 \mathrm{~mm}$.)

Despite their claims to the contrary, the luminous flux account also fails to work for Rubin and Kanwisher's (1985) data, summarized in Figure 2. Columns C and D replicate the results of Chen (1982), while columns A and $B$ constitute new data. Rubin and Kanwisher observe that the difference in black area within stimulus pairs is monotonically related to the proportion of correct responses, and claim that this supports the luminous flux account. They neglect to observe, however, that there is no systematic correspondence between significant differences in the variable to be explained-proportion correct-and the variable they claim is responsible-black area differences.

As Table 1 shows, there is no meaningful luminous flux difference between Pairs B and C, but there is a sizable difference in discriminability. ${ }^{2}$ There is a much larger luminous flux difference between Pairs $A$ and $B$, but no meaningful difference in performance. Only in comparing $C$ and $D$ do we see agreement between meaningful luminous flux difference and performance--but this is exactly the prediction of the topological account. Columns $A$ and $B$ cannot properly be explained by either the luminous flux or the topological account. In Section 2, I will return to this issue, and show that when these stimuli

Table 1

Differences in Black Areas and in Correct Responses Across the Stimulus Pairs in Figure 2

\begin{tabular}{lccc}
\hline & \multicolumn{3}{c}{ Stimulus Pairs } \\
\cline { 2 - 4 } \multicolumn{1}{c}{ Difference in: } & B-A & C-B & D-C \\
\hline Black area $\left(\mathrm{mm}^{2}\right)$ & 92 & 4 & 158 \\
Corresponding responses $(\%)$ & 1.5 & 9.3 & 19.1 \\
\hline
\end{tabular}


are improved to remove confounding effects, the corresponding results can be explained by the topological, but not the luminous flux, account.

In addition to luminous flux, several other nontopological factors have been proposed as counterexplanations to the topological account. These factors include perimeter length, spatial frequency, and the number of edges crossed while scanning the figure. I will now describe Experiments 1 and 2, which were designed specifically to rule out these counterexplanations for discriminations between figures with different numbers of holes.

\section{Experiment 1}

\section{Method}

Subjects. Eight undergraduate students, who were volunteers naive to the purpose of the study, participated in the experiment. All had normal or corrected-to-normal vision.

Stimuli. The three stimulus displays shown in Figure 3 were used in the experiment. These stimulus pairs were drawn in black ink on white cards. Stimulus A contained a disk and a solid square located at either side of the central fixation point, and the distance between the nearest points of the disk and the solid square was $26 \mathrm{~mm}$. The diameter of the disk $(38 \mathrm{~mm})$ was the same as the length of one side of the square. Stimulus B contained a ring and an Slike figure with the same area as that of the ring. The distance between the S-like figure and the ring was also $26 \mathrm{~mm}$. The outer diameter of the ring was the same as that of the disk in Stimulus A $(38 \mathrm{~mm})$, and the inner diameter was $20 \mathrm{~mm}$. The S-like figure was made to approximate the area of the ring, and its shape was purposely made irregular, as shown in Figure 3, in order to eliminate the possible effects of subjective contours or other organizational factors (such as parallelism, or similarity of length). The S-like figure and the disk contained in Stimulus $C$ were the same as the S-like figure in Stimulus B and the disk in Stimulus A, respectively.

Procedure. A three-field Gerbrand tachistoscope, $90 \mathrm{~cm}$ in length, was used to present the stimulus displays. The subjects looked at the fixation point on a preexposure field and pressed a button to receive a 5-msec presentation of a field containing one

A
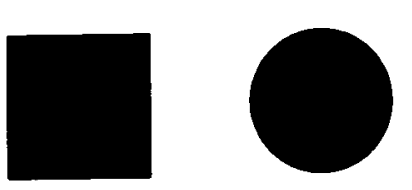

$\begin{array}{cc}\% & \text { Area } \\ \text { correct } & \text { difference } \\ \text { response } & \left(\mathbf{m m}^{2}\right)\end{array}$

$35.6 \%$

310

$\mathbf{B}$
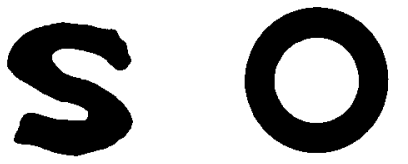

$\mathbf{5 9 . 0 \%}$

0
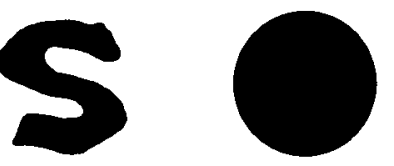

$41.1 \%$

314

Figure 3. The three stimulus displays used in Experiment 1. Also shown are the mean correct response and the area difference for each stimulus pair. of the three stimuli, followed by the immediate reappearance of the preexposure field. The subjects were asked to report whether the two figures in one display were the same or different. (In fact, the displays were always different.) The intensity of illumination of the preexposure field was fixed, and the intensity of illumination of the target field was adjusted at the beginning and might be adjusted further between blocks of presentation for each subject to keep an overall probability of reporting "different" at about $50 \%$. Four blocks of 12 trials per block, each of which contained 4 presentations of each stimulus display, were used as test presentations. The order of presentations was randomized and counterbalanced across subjects.

\section{Results and Discussion}

In Figure 3, on the average, the stimulus (Figure 3A) that contained a disk and a solid square was reported as "different" on $35.6 \%$ of occasions; the stimulus (Figure 3C) that contained a disk and an S-like figure, on $41.1 \%$; and the stimulus (Figure 3B) that contained a ring and an S-like figure, on $59.0 \%$. The shapes of Stimulus B were correctly reported as different significantly more often than those of Stimuli $\mathrm{A}$ and $\mathrm{C}[t(7)=$ $11.11, p<.001$, and $t(7)=3.451, p<.01$, respectively]. For the topologically equivalent figures (Stimuli $A$ and $C$ ), the difference in accuracy did not reach significance $[t(7)=1.078, p>.3]$.

Let us consider the various explanations that have been proposed for these data, starting with luminous flux. The difference in area between the figures in the stimulus pairs is 0 for Pair B, and it is approximately $310 \mathrm{~mm}^{2}$ for Pairs $A$ and $C$. The luminous flux prediction is therefore that B should be less discriminable than the other two casesexactly the reverse of the data.

If discriminability is to be explained by the difference in perimeters between the paired stimuli, the prediction is that $\mathrm{C}$ is more discriminable than both $\mathrm{A}$ and $\mathrm{B} .{ }^{3}$ In fact, however, $\mathrm{C}$ is less discriminable than $\mathrm{B}$, and not significantly more discriminable than $\mathrm{A}$.

It is commonly claimed with respect to the stimuli of Figure 1 that the ring-disk pair is the most discriminable because the ring has stronger high spatial frequency components than the solid figures have. Experiment 1 shows the inadequacy of this proposed explanation. Because the high spatial frequency components of the S-like figure are greater than those of the ring (the length of edge of the S-like figure is greater than that of the ring, and the contour of the S-like figure is irregular) and the high spatial frequency components of the ring are greater than those of the disk, high spatial frequency differences are greater for Pair $C$ (the difference between the S-like figure and the disk) than for Pair B (the difference between the Slike figure and the ring). This spatial frequency account predicts greater discriminability for $\mathrm{C}$ than for $\mathrm{B}$. The data show that exactly the reverse is true.

The account of discriminability based on different numbers of edges crossed while the figures are scanned is complicated by the fact that the number of edges crossed will depend in general on the line of scan; this measure is not really well-defined. For the solid disk and square, there are two crossings, but for the S-like figure, there can be 
two, four, or six crossings, and for the ring, two or four crossings. It would thus appear that this account predicts greatest discriminability for $\mathbf{C}$, and least for $\mathbf{A}$. In fact, $\mathrm{C}$ and $\mathrm{A}$ are equally discriminable-it is $\mathrm{B}$ that is most discriminable.

In fact, for simple figures like disks and rings there is a correlation between the number of holes and the number of edge crossings. In this experiment, we have decoupled the two factors and shown that it is the topological property-the number of holes-and not its correlate (the number of edge crossings) that provides the correct account of early discriminability.

Finally, while luminous flux, perimeter length, spatial frequency, and edge crossings all make wrong predictions for these data, the topological account gets the results exactly right: The only pair in which the two figures are not topologically equivalent is B: the ring has one hole and the S-like figure has none. This gives a direct explanation for why $B$ is the most discriminable among these pairs, and why $A$ and $C$ do not differ significantly in discriminability.

\section{Experiment 2}

According to the topological hypothesis, early discriminability should reflect topological distinctions such as the number of holes in a figure. Preceding experiments have tested the difference between no holes and one hole; this is just one special case of the more general topologi$\mathrm{cal}$ invariant of the number of holes. Experiment 2 was primarily designed to test the more general case by considering the difference between one and two holes. As in Experiment 1, the stimuli were also designed to control for luminous flux and perimeter length.

\section{Method}

Subjects. Eight subjects with the same requirements as those in Experiment 1 participated in the experiment. None of them had participated in Experiment 1.
Stimuli. Two pairs of stimulus displays (shown in Figure 4) were used in the experiment. The pair in Figure 4B was composed of a black disk with one hole and another black disk with two holes. To control for luminous flux, the outer diameters of the two disks were the same $(38 \mathrm{~mm})$, and the sum of areas of the two small holes was designed to be the same as that of the one large hole in the other figure. So, the diameter of each small hole was equal to $\sqrt{2} / 2$ diameter of the large hole $(20 \mathrm{~mm})$. The other pair, in Figure 4A, was similar to Stimulus $C$ in Figure 2, used by Rubin and Kanwisher, except that the diameter of the disk ( $38 \mathrm{~mm}$ ) and the length of one side of the solid square $(38 \mathrm{~mm}$ ) were made longer than those of Stimulus $\mathrm{C}$ in Figure 2, in order to ensure that the two smaller holes within the disk in Figure 4B, the diameter of which was the same as that of the disk in Figure 4A, would be big enough to be visible for a very brief presentation.

Procedure. A procedure similar to that in Experiment 1 was used in Experiment 2.

\section{Results and Discussion}

The percentage of correct identification for Stimulus B (66.2\%) was significantly higher than that for Stimulus A $(39.0 \%)[t(7)=3.809, p<.01]$.

The difference in areas for Pair A is $310 \mathrm{~mm}^{2}$, while it is 0 for Pair B. The perimeter difference for $A$ is $33 \mathrm{~mm}$ $(4 \times 38-\pi \times 38)$, which is greater than that for $B, 26 \mathrm{~mm}$ $(\pi \times \sqrt{2} \times 20-\pi \times 20)$. Thus the luminous flux and perimeter accounts both make the wrong prediction. The topological account makes the correct prediction, since Pair A involves two topologically equivalent figures (both have no holes), while Pair B involves topologically inequivalent figures (one has a single hole and the other two holes).

This experiment provides support for the more general version of the topological hypothesis with respect to holes: Early discrimination is sensitive to the number of holes. This strengthens the challenge to the computational approach to vision, for the computational theory must not only account for early discrimination of a binary-valued property, such as having holes versus not having holes; it must account for discriminations among numbers of holes, requiring still further computational complexity.

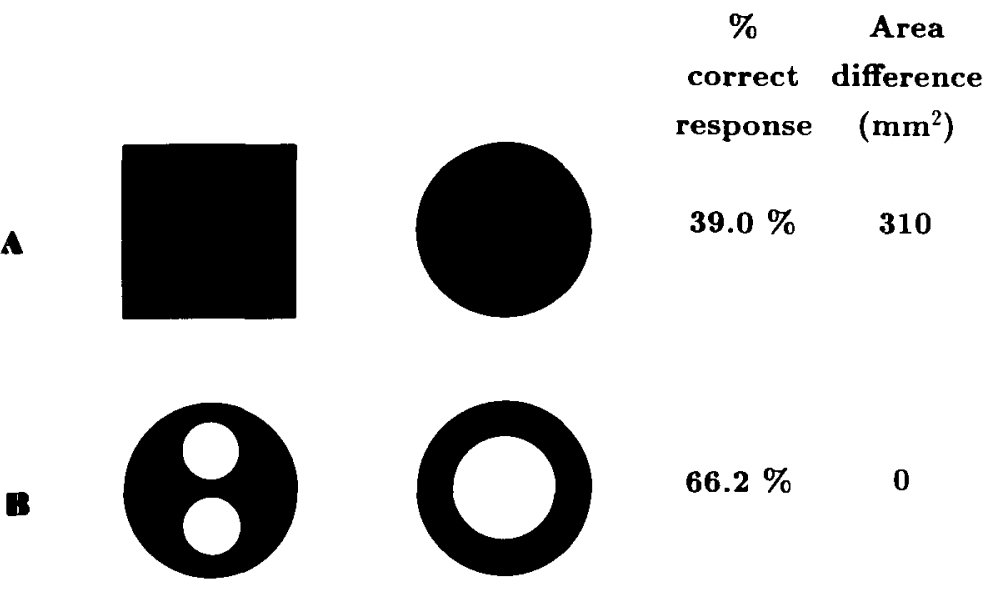

Figure 4. The two stimulus displays used in Experiment 2. Also shown are the mean correct response and the area difference for each stimulus pair. 


\section{ELIMINATING CONFOUNDS IN TWO APPARENT COUNTEREXAMPLES}

\section{Experiments 3 and 4}

Although Experiments 1 and 2 produced the results predicted from a topological analysis and ruled out the alternative explanation of luminous flux, there remains the problem raised in Figures 2A and 2B. Apparently, under normal viewing conditions, the two stimulus pairs are topologically different, but both of them are no more discriminable than the disk-square pair. It seems that despite their failing to support the luminous flux explanation, these data still serve as counterexamples against the topological hypothesis.

In their study of perceptual organization, Pomerantz, Sager, and Stoerer (1977) differentiated the concept of visibility from the concept of discriminability: "Most likely it was the small visual angles of the stimuli that were responsible for the long RTs: The stimuli were simply difficult to see. Nevertheless, it is possible that the difficulty of the discrimination and not the visibility of the stimuli was responsible, that is, that performance was process rather than state limited [emphases added]." Since our visual resolution is not perfect, there must necessarily be cases in which performance errors are mainly caused by stimuli that are simply difficult to see: Arbitrary impoverishment of visual conditions, as in the reducing of visual angles of stimuli and the increasing of the distance of targets from fixation, will make their distinction arbitrarily difficult. Particularly, for very brief presentations, visibility (or the least noticeable difference) will be worse (or bigger): A difference in a stimulus display that may be visible under normal visual conditions may become less perceptible. So, a key purpose in designing the stimulus figures in Figure 1 was to enable a study of the difficulty of discrimination of stimulus patterns while keeping the visibilities for the two figures studied as similar as possible. (The pairs of stimulus figures possess a symmetrical structure with respect to the fixation point.) However, in arguments against topological perception, as often as not, it happens that the concept of visibility is ignored or confused with the concept of difficulty of discrimination. The stimulus displays shown in Figures $2 \mathrm{~A}$ and $2 \mathrm{~B}$ seem to be examples that represent this sort of confusion. The two holes in Stimulus A were located at different distances from the fixation point. Although, under normal visual conditions, both of the two holes and the thin bar between the two holes are visible, for a brief presentation with masking, the upper left hole and the thin bar may be less visible. The gap of the horseshoe (Figure 2B) was located at the bottom position, farther away from the fixation point, and may not be visible for a brief tachistoscopic presentation. So, the two following experiments were specially designed to improve the experimental conditions, taking due consideration of the factor of visibility.

As is mentioned in the literature on tolerance spaces (Chen, 1983, 1985; Zeeman, 1962), given a certain set of conditions, there is always a tolerance within the range of which a difference of stimulus information will be ignored by the visual system because it is less than the least noticeable difference (or visibility) or because it is too small an effect for attaching importance to global organization. (In this case, other organizational factors may dominate global organization, as will be considered further in the next discussion section.)

\section{Method}

Subjects. There were 9 subjects in Experiment 3, and 7 subjects in Experiment 4. The subjects all met the same requirements as those in Experiments 1 and 2. None of them had participated in Experiments 1 or 2.

Stimuli. For Experiment 3, the three stimulus displays shown in Figure 5 were employed. Two of them were exactly the same as Stimuli $\mathrm{C}$ and $\mathrm{D}$ in Figure $2{ }^{4}$

The third one was similar to Stimulus $\mathrm{A}$ in Figure 2, except that the two holes in that stimulus were now rotated in order to be located at the same distance from the fixation point, and the bar between the two holes was widened to $4.5 \mathrm{~mm}$ to improve the visibility of the two holes.

For Experiment 4, another three stimulus displays were presented. Two of them were the same as Stimuli C and D in Figure 2. The third one was the same as Stimulus B in Figure 2, except that the horseshoe was rotated clockwise through $90^{\circ}$ to make its gap open directly toward the fixation point. In this case, the gap became more visible, as shown in Figure 6.

Procedure. The procedure in the experiments was similar to the procedure in Experiments 1 and 2.

\section{Results and Discussion}

With the improvement of visibility, the results turned out to be evidence for the suggested topological structure in visual perception. In Experiment 3, both Stimuli $A$ and $C$, representing discrimination based on the number of
$\mathbf{A}$
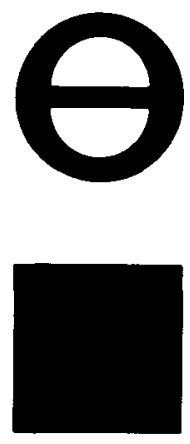

$\mathbf{B}$

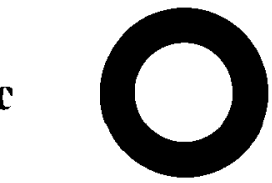

$\%$

correct

response

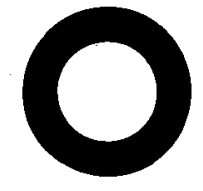

$63.4 \%$

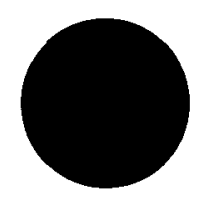

$48.5 \%$

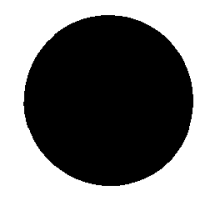

$67.6 \%$
Figure 5. The three stimulus displays used in Experiment 3. Also shown is the mean correct response for each stimulus pair. 
$\mathbf{A}$
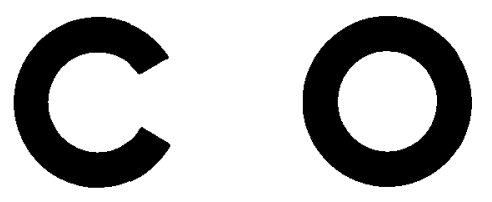

response

$67.6 \%$

$47.4 \%$
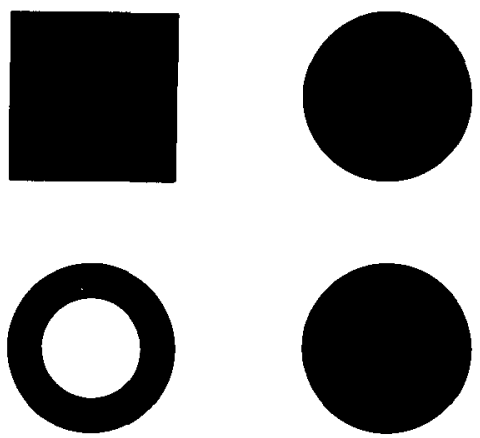

$75.0 \%$

Figure 6. The three stimulus displays used in Experiment 4. Also shown is the mean correct response for each stimulus pair.

holes, demonstrated high percentages of correct identification (63.4\% and $67.6 \%$, respectively), and the difference between these two stimulus displays did not reach significance $[t(8)=1.34, p>.2]$; Stimulus B, representing discrimination in the absence of a topological difference, produced a low discrimination (48.5\%) and was significantly less discriminable than both Stimuli $\mathbf{A}$ and $\mathrm{C}$ $[t(8)=3.00, p<.02$, and $t(8)=5.80, p<.001$, respectively].

Similar results to those of Experiment 3 were obtained in Experiment 4; The horseshoe-annulus pair and the disk-annulus pair, representing discrimination based on topological difference, gave high percentages of correct identification (67.6\% and $75.0 \%$, respectively), both of which were significantly higher than that with the disksquare pair $(47.4 \%)[t(6)=2.62, p<.05$, and $t(6)=$ $5.095, p<.01$, respectively]. It was also found that the two pairs that involved discrimination based on the same topological difference (A and $\mathrm{C}$ ) did not differ significantly $[t(6)=0.871, p>.4]$.

In addition to the concept of visibility and the concept of difficulty of discrimination as differentiated above, one more pair of concepts makes topology in tolerance spaces rather than general topology relevant to visual perceptual organization (Chen, 1983, 1985). In the arguments against the topological hypothesis, people often choose figures with special percepts such as two halves of a circle close to each other and a horseshoe-like figure, as in Stimuli A and B in Figure 2, probably because, psychologically, two halves of a circle close to each other look like one circle, and a horseshoe looks like an annulus. The interesting question is: Although they are mathematically very different, why do they look like each other? It is well- known in perceptual organization that, in addition to physical (spatial) connectedness, other organizational factors (such as similarity) make stimulus figures perceived as being connected or as having holes (see the account of tolerance connectedness and tolerance holes in Chen, $1983,1985)$. For all the experiments reported above, the geometrical properties determined by physically spatial relations were the primary focus of study, in order to reveal the influence of topological factors based on physical (spatial) connectedness under controlled conditions, other organizational factors being eliminated as much as possible. Out of consideration for differentiating organization based on physical connectedness from that based on other factors, the two holes in Stimulus B of Figure 4 are two smaller circles, rather than the two halves of a bigger circle with their diameters close to each other in Stimulus A of Figure 2, in which other organizational factors, such as subjective contour, make the two halves of the bigger circle look like one bigger circle; and the irregular S-like figure (for avoiding the effect of subjective contours, for example) was used in Figure 3, rather than the horseshoe-like figure in Stimulus B of Figure 2. Although Experiments 1 and 2 had been run before the experiments of Rubin and Kanwisher (1985), and Experiments 3 and 4 were particularly designed to deal with the issue raised by their experiments, Experiments 1 and 2 may be functionally considered as improvements of Experiments 4 and 3, respectively, and indeed strengthened the early topological perception viewpoint. As the data of these experiments show, the difference in percentages of correct identification between Stimuli A and B in Experiment 1 is greater than that in Experiment 4 [23.4\% and $t(7)=11.11, p<.001$, and $19.6 \%$ and $t(6)=2.63$, $p<.05$, respectively], and the difference between Stimuli $A$ and $B$ in Experiment 2 is also greater than that in Experiment $3[27.2 \%$ and $t(7)=3.809, p<.01$, and $14.9 \%$ and $t(8)=3.00, p<.02$, respectively].

\section{GENERAL DISCUSSION}

In their article, Rubin and Kanwisher (1985) make two points: (1) that the data from Chen (1982) and their own new data are consistent with a luminous flux account, and (2) that the new data are inconsistent with the topological account. With respect to the first point, I have shown that when certain rather prominent aspects of their data are considered rather than ignored, the data are seen not to be consistent with a luminous flux account. Furthermore, Experiments 1 and 2 reported here reinforce previous studies in showing the inadequacy of the luminous flux explanation as well as several other nontopological counterexplanations. With respect to Rubin and Kanwisher's second point, I argue that the reason why their data is inconsistent with the topological account is that the stimuli were confounded by a failure to take due account of two factors: visibility, and other organizational factors besides physical connectedness. Both of these factors have been extensively discussed previously (Chen, 
1983, 1986), and it is not difficult to design stimuli to avoid these confounds. Improving Rubin and Kanwisher's stimuli mainly to eliminate the visibility confound leads to Experiments 3 and 4; the results are completely consistent with the topological account (and inconsistent with the luminous flux account). Further improving the stimuli to eliminate the confound of other organizational factors leads in fact back to Experiments 1 and 2, in which the topological effects are even stronger.

The topological hypothesis provides a strong challenge to computational approaches to vision based on local geometrical primitives. The experiments reported here further strengthen the empirical basis for this challenge.

\section{REFERENCES}

Chen, L. (1982). Topological structure in visual perception. Science, 218, 699-700.

CHEN, L. (1983). What are the units of figure perceptual representation? (Studies in Cognitive Science No. 22). Irvine, CA: University of California, Irvine, School of Social Sciences.

ChEN, L. (1985). Topological structure in the perception of apparent motion. Perception, 14, 197-208.

Chen, L. (1986). Topological perception-a possible dark cloud over computational theory. In Qian Xue Sen (Ed.), Essays on cognitive sciences (pp. 250-301). Shanghai: People's Press of Shanghai. (In Chinese)

KLüVER, H. (1941). Visual functions after removal of the occipital lobes. Joumal of Psychology, 11, 23-45.
Minsky, M. L., \& PAPERT, S. (1969). Perceptrons: An introduction to computational geometry. Cambridge, MA: MIT Press.

Pomerantz, J. R., Sager, L. C., \& Stoerer, R. J. (1977). Perception of wholes and of their component parts: Some configural superiority effects. Journal of Experimental Psychology: Human Perception \& Performance, 3, 422-435.

Rubin, J. M., KANWISHER, N. (1985). Topological perception: Holes in an experiment. Perception \& Psychophysics, 37, 179-180.

ZeEman, E. C. (1962). The topology of the brain and visual perception. In K. Furt (Ed.), Topology of 3-manifolds (pp. 112-114). Englewood Cliffs, NJ: Prentice-Hall.

\section{NOTES}

1. The area of the ring was actually $6.8 \%$ larger than that of the triangle, which, on a luminous flux account, would make it less discriminable from the disk, contrary to the data.

2. The difference, $4 \mathrm{~mm}^{2}$, is so small as to be within the error of drawing figures by hand.

3. The perimeter difference in $C$ exceeds that in $B$ by the perimeter of the inner circle in the ring, $62.8 \mathrm{~mm}$. The perimeter difference in A, $32.7 \mathrm{~mm}$, is less than that in C.

4. The square in Figure 2D has sides of $30 \mathrm{~mm}$. The black diskused to construct all of the circular figures in our experiment-has a diameter of $32 \mathrm{~mm}$. The inner radius of the annulus is $18 \mathrm{~mm}$. The horseshoe in Figure 2B is identical to the annulus except that a $60^{\circ}$ sector is removed. The two holes of the disk-with-two-holes in Figure $2 \mathrm{~A}$ are semicircles of diameter $18 \mathrm{~mm}$. The differences in the black areas of each figure pair are shown in Figure 2.

(Manuscript received November 11, 1988; revision accepted for publication July 18, 1989.) 